\title{
Relational and Item-Specific Encoding Task
}

National Cancer Institute

\section{Source}

National Cancer Institute. Relational and Item-Specific Encoding Task. NCI Thesaurus.

Code C131877.

A clinical test designed to assess episodic memory encoding and retrieval processes in individuals with cog nitive impairment. 